\title{
No association between ischemic heart disease and periodontitis in women
}

\author{
Abstracted from \\ Stenman U, Wennstrom A, Ahlqwist M, et al. \\ Association between periodontal disease and ischemic heart disease among Swedish women. \\ A cross-sectional study. Acta Odontol Scand 2009; 19: 1-7 \\ Address for correspondence: Ulrika Stenman, Folktandvården Lindome, Box 53, \\ SE-437 21 Lindome, Sweden. E-mail: ulrika.stenman@vgregion.se
}

\section{Question: Is there an association between ischemic heart disease and periodontitis in middle-aged and elderly women?}

Design A cross-sectional study was conducted.

Sample A random sample of women was used from the participants in a Prospective Population Study of women in Gothenburg that had been initiated in 1968.

Data sources Dental examinations were conducted by dentists and included panoramic radiography; a clinical inspection of the teeth, gums and oral mucosa; colour photography of the dentition, and a questionnaire. Myocardial infarction was diagnosed if at least two of the following criteria were present: 1) central chest pain $>30$ min; 2) transient rise of transaminase activities; and 3) typical electrocardiogram changes of recent onset. Angina pectoris was diagnosed using a questionnaire. The other independent variables were obtained from the medical part of the study and constituted other well-known risk factors for IHD.

Results Among the dentate women in this study $(\mathrm{N}=847), 74 \mathrm{had}$ ischemic heart disease (IHD) and 773 did not. There was no statistically significant difference between numbers of pathological gingival pockets between these groups $(58.1 \%$ had one or more pathological pockets in the IHD group versus $57.6 \%$ in the non-IHD group). Bivariate analysis of dentate individuals showed significant associations between IHD and number of missing teeth, age, body mass index, waist/ hip ratio, life satisfaction, hypertension, and levels of cholesterol and triglycerides. In the final multivariate logistic regression model, however, with the exception of age, only number of teeth [B17 teeth; odds ratio, 2.13; $95 \%$ confidence interval $(\mathrm{Cl}), 1.20-3.77]$ was found to be significantly associated with IHD. Moreover, edentulous women had an odds ratio of $1.94(95 \% \mathrm{Cl}, 1.05-3.60)$ in relation to IHD (age-adjusted model).

Conclusions In the present study, periodontitis did not seem to have a statistically significant relationship with IHD. The number of missing teeth showed a strong association with IHD, and this may act as a proxy variable tapping an array of different risk factors and behaviours.

\section{Commentary}

Interest continues unabated on the association between oral health conditions and the risk of atherosclerotic heart disease, in both the research and practice communities. In 2009, the American Journal of Cardiology and the Journal of Periodontology, both prestigious professional journals, jointly issued evidence-based clinical recommendations for both medical and dental professionals to use in managing patients with, or who are at risk for, either periodontitis or heart disease. ${ }^{1} \mathrm{~A}$ goal of this effort is for dentists and physicians to enhance the management of heart disease in periodontitis patients and the management of periodontitis in heart disease patients.

In the research literature, there is increasing evidence to support an association between oral conditions and risk of IHD. Two recent meta-analyses have concluded that periodontitis is a risk factor or marker for IHD, independent of other recognised IHD risk factors. ${ }^{2,3}$ Meanwhile, the original report by Stenman et al., which finds no significant association between periodontitis and IHD in a sample of 847 dentate Swedish women, examined in 1992-1993, and drawn from the Prospective Population Study of Women in Gothenburg. Interestingly, whereas periodontal status was not found to be associated with IHD, they did find that tooth loss was significantly associated with IHD. Using a multivariate model adjusting for several IHD risk factors, they found that women with fewer than 17 teeth were 2.13-fold more likely (95\% CI, 1.20-3.77) to have IHD than women who had 17 or more teeth. Moreover, women who were fully edentulous were also at higher odds of having IHD.

Given this significant finding, perhaps this Evidence-based Dentistry Summary could be alternatively titled, "Significant association between ischemic heart disease and tooth loss in women". Such apparently disparate findings, however, should in no way detract from the overall value of this excellent study. The study design and data analyses were well done, given the inherent limitations of the cross-sectional data used. It is important to note that the women here were categorised as having periodontitis if they had one or more "pockets of $6 \mathrm{~mm}$ or more." It is thus unclear the extent to which 'misclassification bias' may have been a factor in their null result regarding an IHD association with periodontitis, resulting from setting the periodontitis threshold at that particular level. For example, this periodontitis case definition may have resulted in there having been a large number of women with multiple pockets of 4 and $5 \mathrm{~mm}$ who became classified as not having periodontitis. As has been noted before, such misclassification bias in this type of analysis will tend to bias results to the null. ${ }^{4}$ It may be informative, 
therefore, to re-analyse these data using a more generally accepted case definition of periodontitis. ${ }^{5}$

Perhaps the most noteworthy aspect of the study is that the significant association the authors reported between tooth loss and IHD confirms others' work. In their meta-analysis, Humphrey et $a l .{ }^{3}$ found that the summary estimate for all coronary heart disease (CHD)/ cardiovascular disease events was 1.34 (95\% CI, 1.10-1.63) for individuals with $0-10$ teeth at baseline, as compared with individuals with 25-32 teeth, indicating a 34\% increased risk in people who have fewer teeth. Bahekar et al. ${ }^{2}$ found a similar risk elevation related to tooth loss in their meta-analysis. One of the original reports used in these meta-analyses, a study conducted in approximately 100000 health professionals in the US, found that baseline number of missing teeth was directly related to CHD events or death, with a suggestion of a dose-response relationship. ${ }^{6}$ In 1993, DeStefano et al. ${ }^{7}$ reported that risk of mortality was significantly elevated in completely edentulous individuals, at a level similar to that seen in dentate persons who had periodontitis. More recently, in 2008, Dietrich and colleagues $^{8}$ reported that tooth loss was significantly associated with risk of IHD in a cohort of adult men. These epidemiologial analyses have typically been multivariate and have attempted to control for many relevant covariates, including tobacco use. Nevertheless, there always remain concerns about the potential effects of residual confounding, in particular from smoking. ${ }^{9}$

What inferences may we appropriately draw from this study? How does the finding of no significant association between periodontitis and likelihood of IHD, as well as their finding of a significant association between tooth loss and IHD relate to other work in this area over the past 2 decades, in particular the significant associations found in recent meta-analyses of prospective cohort or case-control studies? Several causal and noncausal pathways have been postulated to explain the observed association between periodontitis and cardiovascular diseases. ${ }^{10}$ Causal pathways may involve direct and indirect effects of periodontal infection and the inflammatory response, whereas an increased pro-inflammatory susceptibility to both atherosclerosis/ thrombosis and chronic periodontitis would be an alternate, noncausal pathway. Current epidemiological studies are not able to differentiate between these causal and noncausal pathways, however, even with statistical adjustment for all established cardiovascular risk factors. Thus, both causal and noncausal pathways may have a role in the observed associations in this review.
Despite their inherent limitations, an important property of observational epidemiological studies is to provide insights into plausible causal pathways and to inform the design of intervention studies. The finding that tooth loss is associated with an increased risk of IHD does not necessarily plausibly imply that replacement of missing teeth will result in IHD risk reduction. Rather, it may be more plausible to posit that risk factors that may be contributing to both tooth loss and to IHD are the more appropriate points of intervention. Mechanisms involving various inflammatory pathways have been proposed as the most fruitful pathways for targeting interventions aimed at improving oral health for the overarching goal of improving cardiovascular outcomes. ${ }^{11-12}$

\section{Raul I Garcia \\ Department of Health Policy and Health Services Research, and the Northeast Center for Research to Evaluate and Eliminate Dental Disparities, Boston University Henry M Goldman School of Dental Medicine, Boston, Massachusetts, USA}

1. Friedewald VE, Kornman KS, Beck JD, et al. The American Journal of Cardiology and Journal of Periodontology Editors' Consensus: periodontitis and atherosclerotic cardiovascular disease. / Periodontol 2009; 80: 1021-1032.

2. Bahekar AA, Singh S, Saha S, Molnar J, Arora R. The prevalence and incidence of coronary heart disease is significantly increased in periodontitis: a meta-analysis. Am Heart J 2007; 154: 830-837.

3. Humphrey LL, Fu R, Buckley DI, Freeman M, Helfand MJ. Periodontal disease and coronary heart disease incidence: a systematic review and meta-analysis. / Gen Intern Med 2008; 23: 2079-2086

4. Dietrich T, Garcia RI. Associations between periodontal disease and systemic disease: evaluating strength of the evidence. J Periodontol 2005; 76 (suppl.): 2175-2184.

5. Page RC, Eke PI. Case definitions for use in population-based surveillance of periodontitis. J Periodontol 2007; 78 (suppl.): 1387-1399.

6. Hung HC, Joshipura KJ, Colditz G, et al. The association between tooth loss and coronary heart disease in men and women. J Public Health Dent 2004; 64: 209-215.

7. DeStefano F, Anda RF, Kahn HS, Williamson DF, Russell CM. Dental disease and risk of coronary heart disease and mortality. Br Med J 1993; 306: 688-691.

8. Dietrich T, Jimenez M, Krall Kaye E, Vokonas PS, Garcia RI. Age-dependent associations between chronic periodontitis/ edentulism and risk of coronary heart disease. Circulation 2008; 117: 1668-1674.

9. Hujoel PP, Drangsholt M, Spiekerman C, DeRouen TA. Periodontitis-systemic disease associations in the presence of smoking - causal or coincidental? Periodontology 2000 2002; 30: 51-60

10. Jimenez M, Krall EA, Vokonas PS, Garcia RI, Dietrich T. Periodontitis and incidence of cerebrovascular disease in men. Ann Neurol 2009; 66: 505-512.

11. Tonetti MS, D'Aiuto F, Nibali L, et al. Treatment of periodontitis and endothelial function. N Engl J Med 2007; 356: 911-920.

12. Offenbacher S, Beck JD, Moss K, et al. Results from the Periodontitis and Vascula Events (PAVE) study: a pilot multicentered, randomized, controlled trial to study effects of periodontal therapy in a secondary prevention model of cardiovascular disease. J Periodontol 2009; 80: 190-201.

Evidence-Based Dentistry (2010) 11, 20-21 doi:10.1038/sj.ebd.6400706 